\title{
The prevalence of delirium on neurological intensive care unit and stroke unit
}

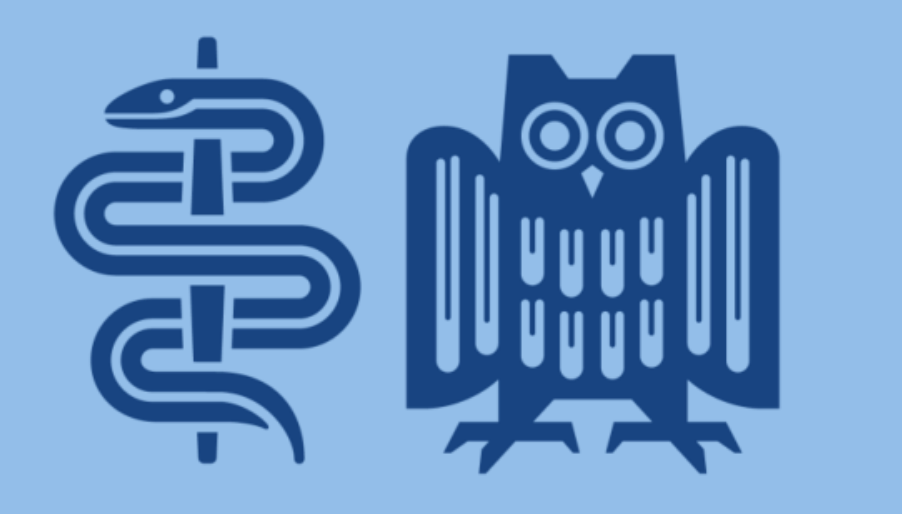

UKS

Universitätsklinikum des Saarlandes

\section{Backround:}

Delirium describes an acute dysfunction of brain activity, presenting as the well-known "confused patient". This is often described after the acute onset of a disease and after surgical interventions. Especially wards with intensified monitoring report about patients in acute mental confusion as part of their daily routine [1].

Many complications come along with these mental confusions, naming prolonged in-patient stay, slowed recovery, higher risk of infection and not to forget the extra effort for both nursing and medical staff.

Many neurological diagnosis requiring intensified care, come with an acute onset and thus are associated with high risk of developing delirium. Other evaluated risk factors for delirium such as high age, dementia, preexistent neurological deficits are also common in stroke units and neurological ICU patients. In the end also the environment and atmosphere of ICU and stroke unit are known to increase the chances of supporting the occurrence of delirium [2] At the neurology department of University Clinic of Saarland patients requiring intensified monitoring and care are treated at a Stroke Unit (16 beds) or a neurology-only Intensive Care Unit ( 8 beds), which are directly connected. Nursing and medical staff at both wards report about higher complication rates, intensified care expenses and higher stress levels due to patients with abnormal psychomotor activity.

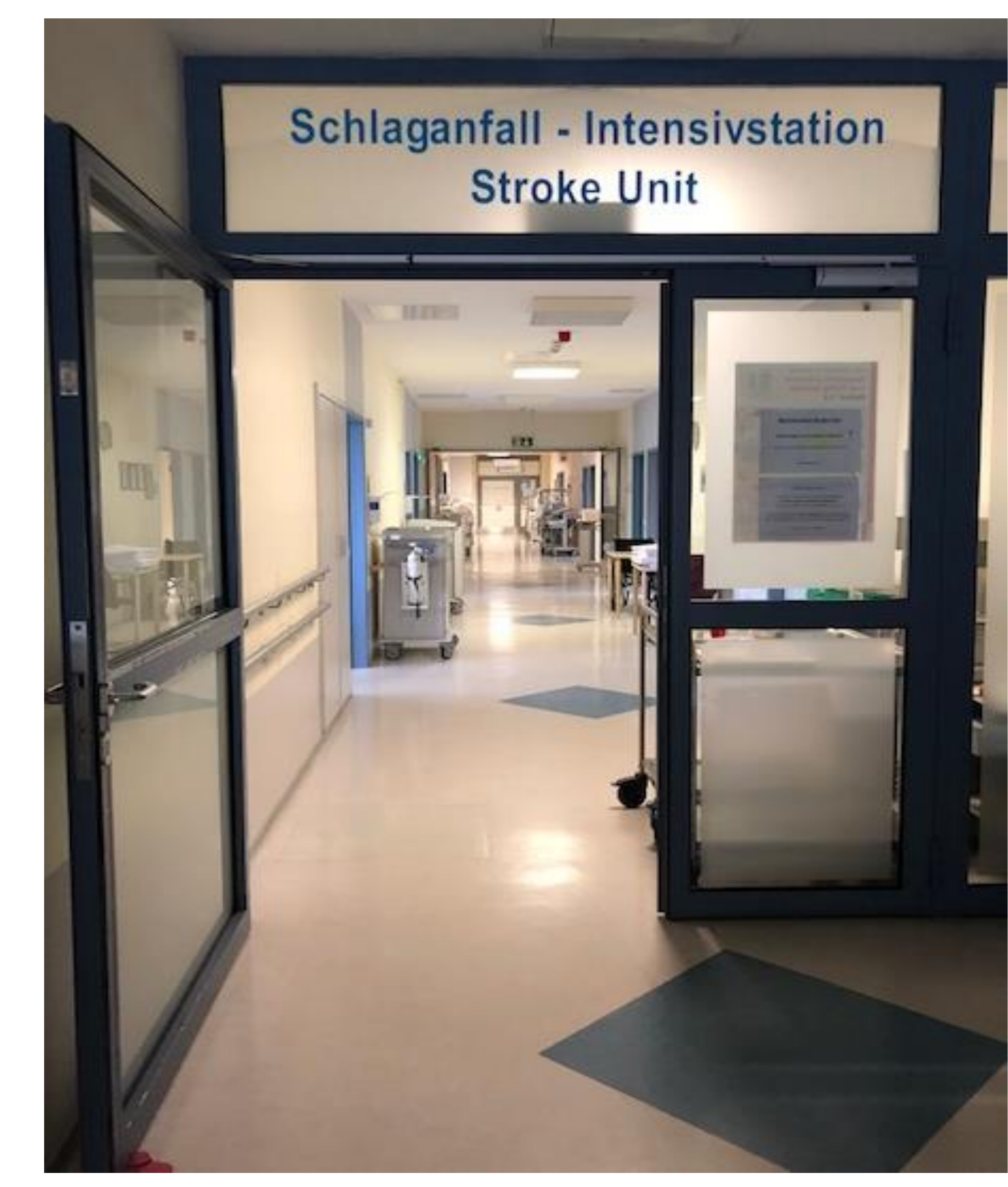

Fig. 1: Stroke Unit

Methods:

During a period of 3 months all patients in the Stroke Unit and Intensive Care Unit were included in the study. At fixed time frames patients were visited throughout their entire stay. Each round the CAM-ICU standardized workflow was carried out and their psychomotor activity was rated by the RASS (Richmond Agitation Scale Score), both being validated methods for delirium screening [3]. Additionally, selected data from the patient chart was noted down

\begin{tabular}{|l|}
\hline CAM-ICU exclusion criteria: \\
- RASS -4 or -5 \\
- Patient sleeping \\
- No way of communication possible (e.g. sensory aphasia) \\
- Language problems
\end{tabular}

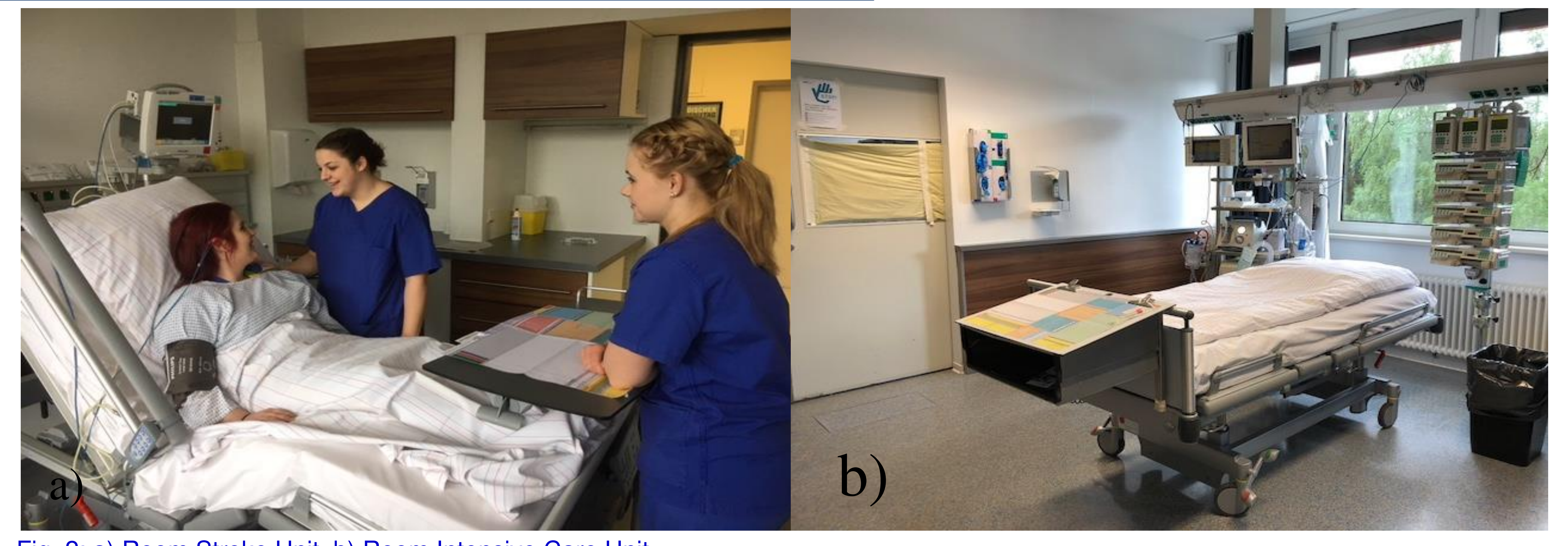

Fig. 2: a) Room Stroke Unit b) Room Intensive Care Unit

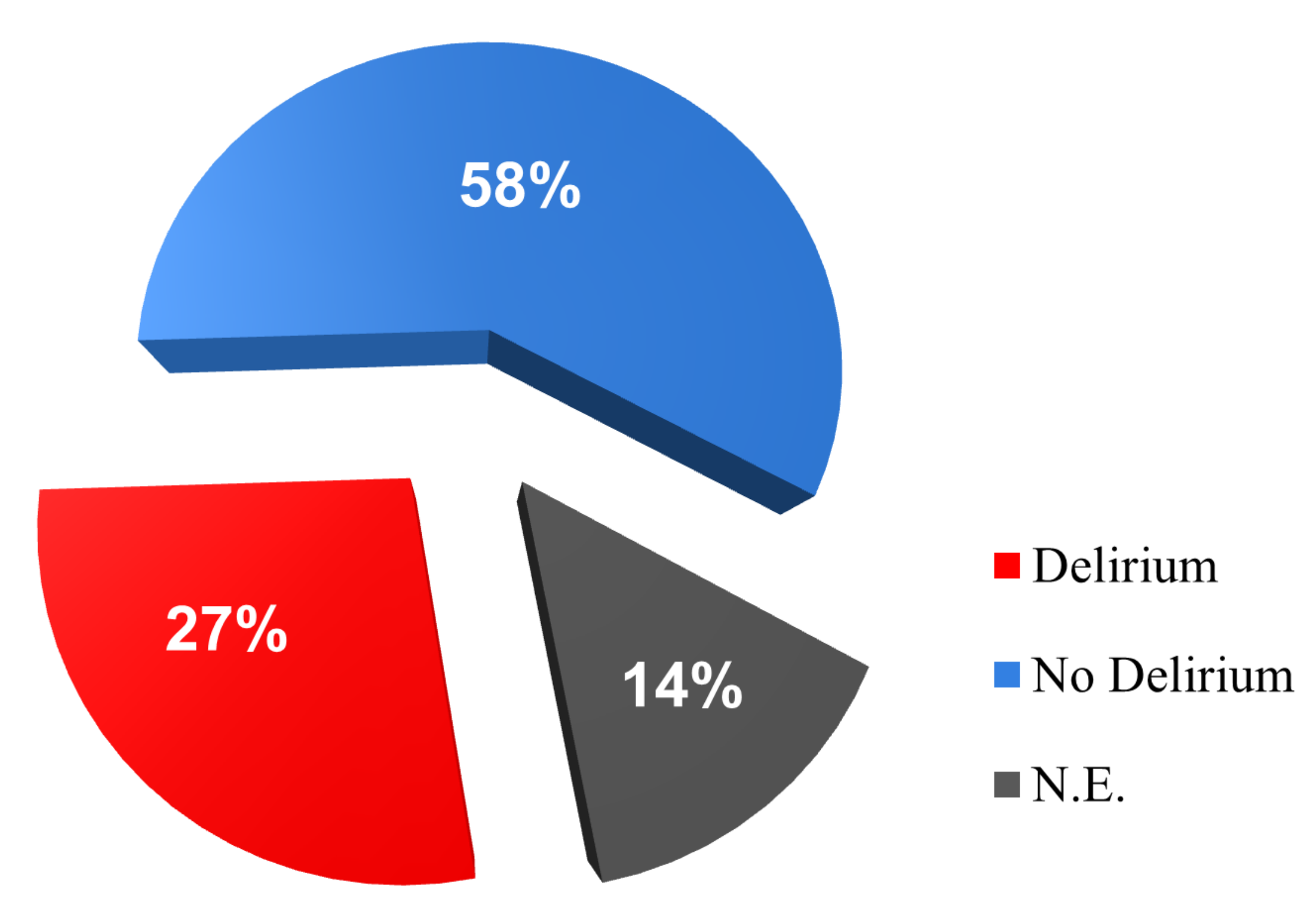

Fig. 3: Delirium prevalence

Results:

An all in all number of 209 patients were included in the study. 128 individuals of the group were diagnosed with having had a stroke or TIA. Other diagnosis were intracerebral hemorrhage, other primarily neurological or non-neurological diagnoses. Evaluating the CAM-ICU 57 patients (27\%) were tested positive for delirium at least one time during their stay, 123 patients (58\%) always showed a delirium negative result and for 29 patients (14\%) the method could not be applied due to exclusion criteria.

In average patients spent 5.08 nights at the units. The average stay of delirium positive patients was 7.16 nights and 3.38 of the delirium negative group.

\section{Case report:}

Patient K.D., 70 year-old man, diagnosed with stroke. The total length of his stay in the Stroke Unit was 8 nights.

Within eight nights and three measurements a day the patient showed a fluctuating RASS (range -2 to +1 ) and a delirium positive CAM-ICU result ten times. Even at times when the RASS value was 0 , K.D. had a positive CAM-ICU for five times. Most of the measurements the patient showed a hypoactive delirium (RASS $<0$ ), once the patient had a hyperactive delirium (RASS $<0$ ).

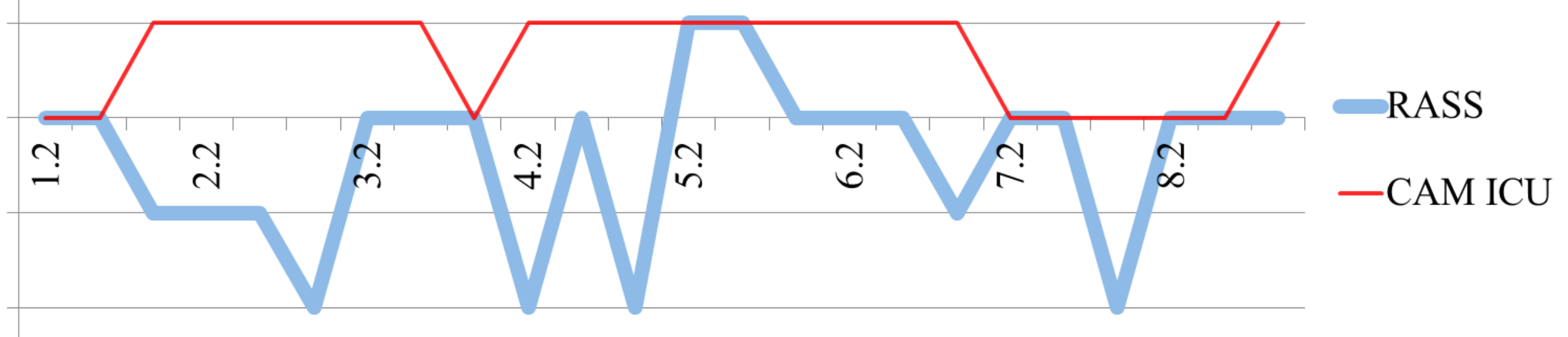

Fig. 4: Patient K.D. RASS and CAM-ICU fluctuation

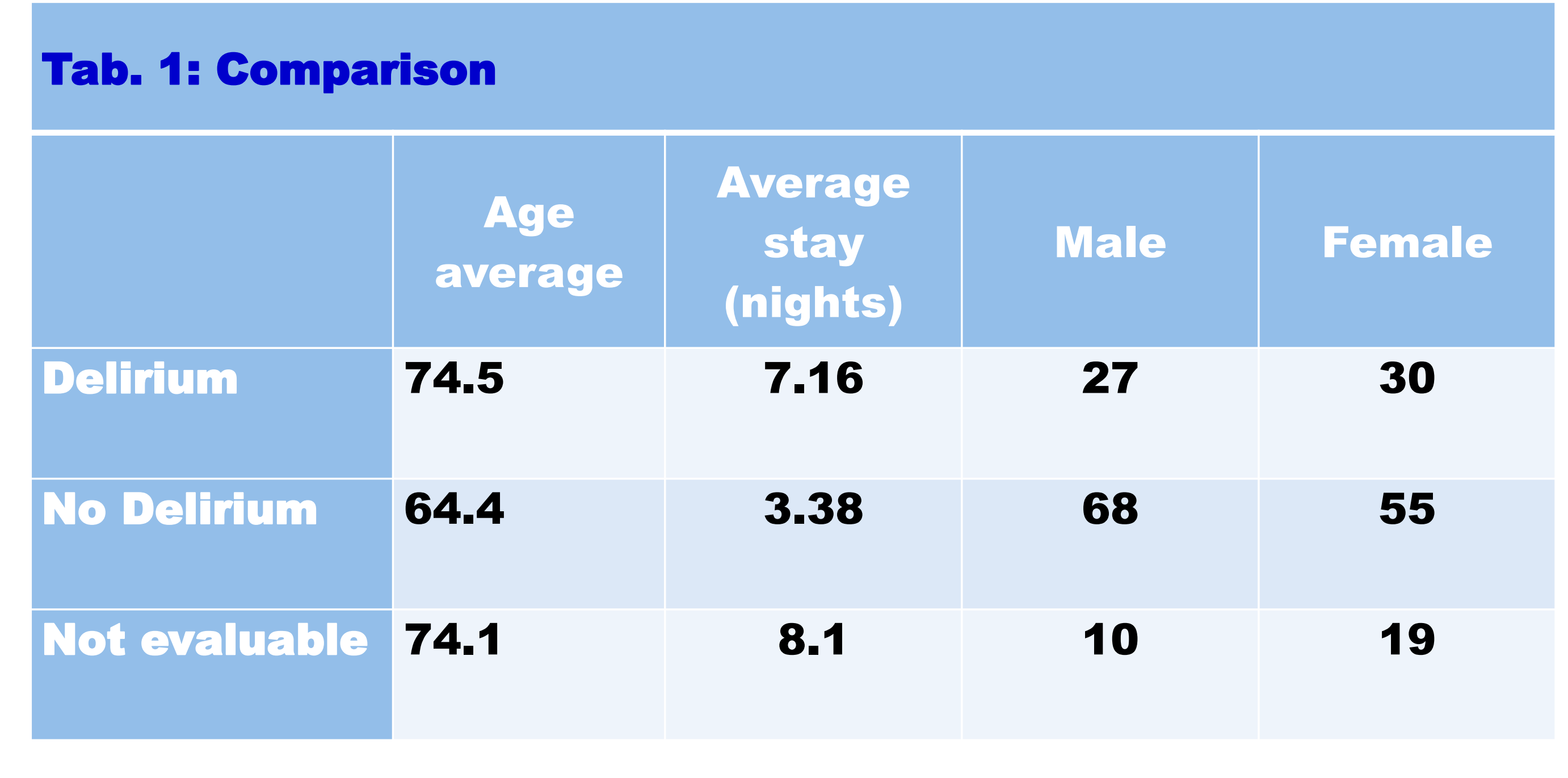

Discussion:

The study could show that delirium is a common complication at Stroke Unit and ICU, which is also leading to longer a hospital stay. The close monitoring allows a better quality of delirium diagnosis and further conclusions about the quality of delirium (hypomotor vs. hypermotor).

The setup of CAM-ICU allows quick and easy bedside testing, but it requires conscious patients with ability to communicate (verbal or by gestures). Therefore exclusion criteria led to $14 \%$ of the group who could never be tested with it. Preexistent neurological deficits and also newly developing neurological symptoms turned out to be the main limiting factor. Furthermore Intensive Care Unit patients under sedation and/or intubation cannot be screened. For those only RASS fluctuation turned out to be a hint for psychomotor instability. But as seen in the case report a positive or a negative RASS is not a safe proof for a present delirium.

In the end this will have to be further validated.

\section{Tab. 2: Patient data $n=209$}

\begin{tabular}{|c|c|}
\hline Age average (range) & $\mathbf{6 8 . 5}(\mathbf{2 0 - 9 5 )}$ \\
\hline Wale & $\mathbf{1 0 5}$ \\
\hline Female & $\mathbf{1 0 4}$ \\
\hline Stroke Unit & $\mathbf{1 8 5}$ \\
\hline IGU & $\mathbf{5 1}$ \\
\hline Stroke Unit \& Gu & $\mathbf{3 2}$ \\
\hline Average stay in nights (range) & $\mathbf{5 ( 0 - 4 8 )}$ \\
\hline Stroke & $\mathbf{1 1 8}$ \\
\hline ThA & $\mathbf{1 0}$ \\
\hline IGB & $\mathbf{8}$ \\
\hline Other neurological diagnosis & $\mathbf{3 0}$ \\
\hline Non neurological diagnosis & $\mathbf{1 5}$ \\
\hline Elective & $\mathbf{2 2}$ \\
\hline Death & $\mathbf{6}$ \\
\hline
\end{tabular}

Abbreviations: ICU= Intensive Care Unit; RASS = Richmond Agitation Scale Score; CAM-ICU = Confusion Assessment Method for ICU; N.E. $=$ not evaluable 